\title{
Research on the Stability of Multi PV Inverters Connected to Weak Grid Based on Impedance Analysis
}

\author{
Wang Qinghao ${ }^{1}$, Ye Dewu ${ }^{1}$, Liu Bo ${ }^{1}$, Qu Yi ${ }^{1}$, Liu Jing ${ }^{2}$, Wang Yi ${ }^{3}$, Liu Guobin ${ }^{1}$, Song Yuanlin ${ }^{1}$, \\ Chen Hui ${ }^{1}$, Quo Junping ${ }^{1}$ \\ ${ }^{1}$ Fushun Power Supply Company, Liaoning Electric Power Company Limited, State Grid, China \\ ${ }^{2}$ Tieling Power Supply Company, Liaoning Electric Power Company Limited, State Grid, China \\ ${ }^{3}$ Measurement Cente, Liaoning Electric Power Company Limited, State Grid, China
}

Keywords: PV inverter, equivalent impedance, phase locked loop design, power system stability

\begin{abstract}
In paralleled grid connected PV inverters system, when the design of the inverter control system is connected with the weak grid, the control performance will become poor, and even the operation is not stable. In this paper, a method of impedance analysis is presented, and the equivalent impedance of inverter is derived by using the small signal model of the inverter, which is used to analyze the stability of inverter parallel system. And this paper proposes that system can be stabilized by changing the phase locked loop design of the inverter. Simulation results verify the validity and feasibility of the theoretical analysis and the proposed method.
\end{abstract}

\section{Introduction}

With the continuous reduction of the reserves of non-renewable energy such as oil and coal, and the improper exploitation and utilization, the energy crisis is going to happen soon. Solar energy, as a sustainable energy alternative, has been developing rapidly in recent years ${ }^{[1] .}$ In recent years, the capacity of photovoltaic power generation system is becoming larger and larger, but the capacity of the photovoltaic inverter is limited. Therefore, the large photovoltaic power plant usually adopts a typical structure of parallel operation of multiple photovoltaic power units. In actual production and construction, with the increase of PV power station capacity, the equivalent impedance of PV system decreases under the restriction of land resources and light resources ${ }^{[2]}$. When the equivalent impedance is reduced to the same as that of the grid impedance, the characteristics of the weak grid will appear in the grid ${ }^{[1]}$. The longer the transmission line and the larger the capacity of PV power station, the stronger the voltage fluctuation caused by output fluctuation and voltage disturbance of PV power station, which will have a great impact on the stable operation of multiple parallel grid-connected PV inverters.

The interaction between inverter and power grid will make the system unstable. For example, the high impedance of the grid will cause the current control loop to be unstable and cause harmonic resonance or instability. Improper design of controller parameters of photovoltaic inverter system will also lead to instability of the inverter output power. Aiming at the problem of system instability caused by the multi photovoltaic inverters connecting to weak grid ${ }^{[4]}$. In this paper, an analysis method based on impedance is proposed. The equivalent impedance of the inverter is derived through the small signal model of the inverter in dq coordinates. The stability analysis of the inverter parallel system is carried out according to the system stability criterion of the automatic control theory. Finally, a solution to adjust the inverter phase locked loop (PLL) is proposed to make the system stable ${ }^{[5]}$.

\section{The stability of multiple photovoltaic inverters in parallel operation}

\subsection{Single inverter grid-connected system structure}

In order to analyze the stability of multi inverter parallel power generation system, a small signal equivalent circuit model is established based on the double loop control structure of the single 
inverter. Figure 1 shows the structure of a single phase grid-connected inverter. The upper part of the diagram is power circuit configuration, and the second half is inverter control structure. $I_{p v}$ is the current output of a photovoltaic array, $\mathrm{U}_{\mathrm{dc}}$ the capacitance voltage of the DC side, $\mathrm{I}_{\mathrm{dc}}$ is the current injecting into inverter, $\mathrm{u}_{\mathrm{abc}}$ is the inverter intermediate point voltage, $\mathrm{L}$ and $\mathrm{R}_{\mathrm{L}}$ is the filter inductance and resistance, $\mathrm{u}_{\mathrm{g}}$ and ig ${ }_{\mathrm{abc}}$ is the node voltage and current, $\mathrm{R}_{\mathrm{g}}$ and $\mathrm{L}_{\mathrm{g}}$ is the resistance and inductance of the grid, $\mathrm{u}_{\mathrm{s}}$ is the grid voltage. The structure of the control system includes the maximum power point tracking (MPPT) control link and power control link.

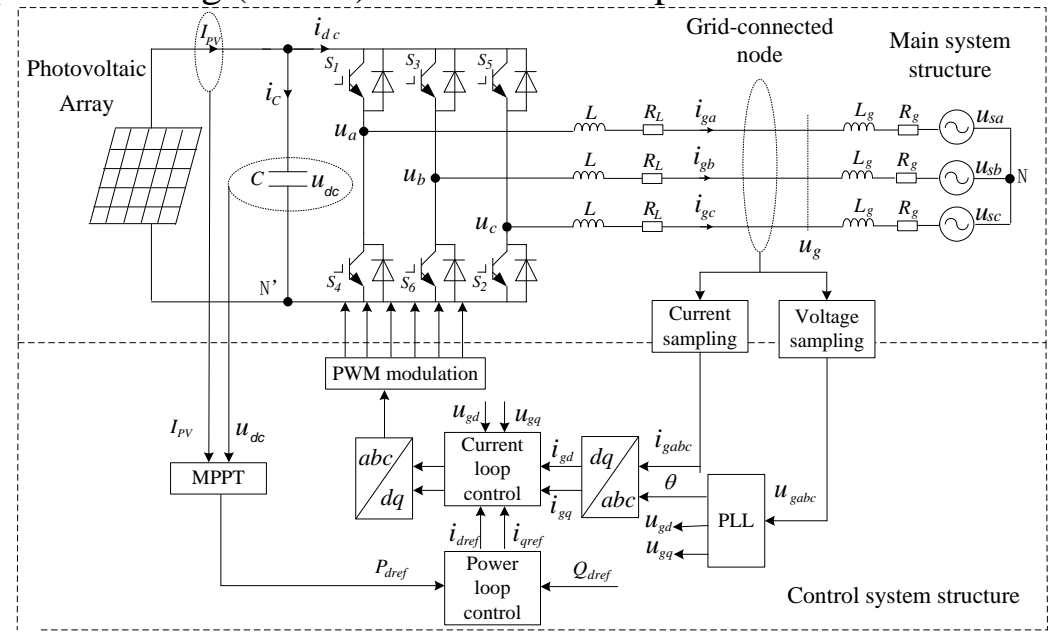

Fig 1 photovoltaic inverter system and control structure

\subsection{Instability of different phase locked loop parameters}
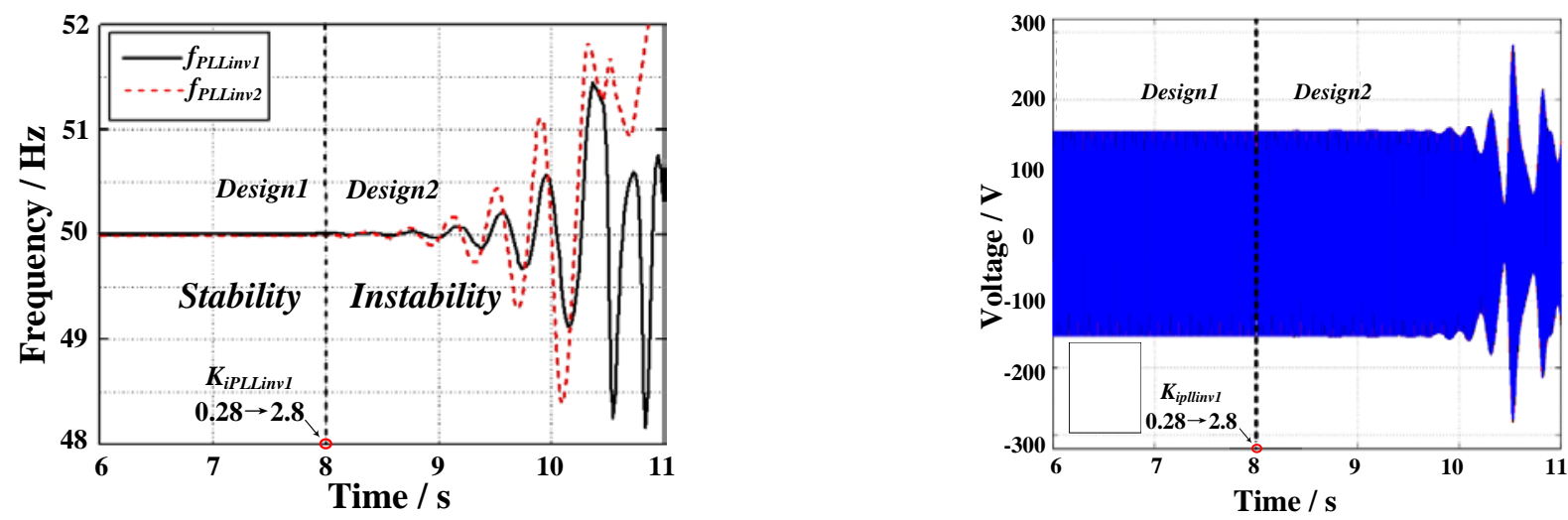

Fig. 2 The output frequency of PLL and common node voltage

The above is the simulation results of two inverters parallel system. It can be seen that in figure 2 , the $\mathrm{k}_{\mathrm{i}}$ parameter of one inverter PLL PI link was changed from 0.28 to 2.8 in 8 seconds, and the frequency of the PLL oscillates, and the frequency of the another PLL was also oscillated. In figure 4, the voltage of the grid-connected node became instability after the PLL parameters change.

\section{Modeling of photovoltaic inverter system}

\subsection{Impedance stability criterion for photovoltaic inverter system}

As shown in Figure 3, the equivalent circuit of the inverter, the inverter can be regarded as a current source $\left(I_{s}\right)$ parallel output impedance $\left(Z_{\text {inv }}\right)$, the grid can be considered as an ideal voltage source $\left(U_{g}\right)$ series grid impedance $\left(Z_{\text {grd }}\right)$.

Based on the equivalent circuit of the inverter shown in Fig. 3, the grid current equation (1) can be derived. It is assumed that the grid voltage is stable when no load exists, and the output of the inverter is stable when connecting to the ideal power grid. According to the linear control theory, so the stability of the output current depends on $\mathrm{Z}_{\text {grd }}(\mathrm{s}) / \mathrm{Z}_{\text {inv }}(\mathrm{s})$. If the ratio of the grid impedance to the 
inverter output impedance satisfies the Nyquist stability criterion, the grid-connected inverter can operate steadily in the grid, and the stability margin of the grid system can be expressed as by the Nyquist curve of $Z_{\text {grd }}(s) / Z_{\text {inv }}(s)$.

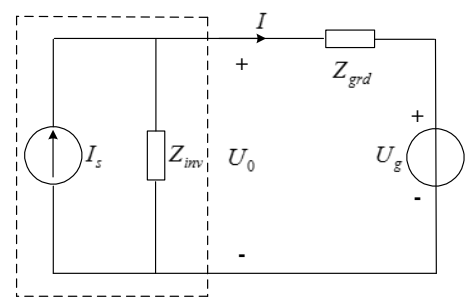

Fig. 3. The equivalent circuit of inverter system based on impedance analysis

$$
I_{g}(s)=\left[I_{s}(s)-\frac{U_{g}(s)}{Z_{\text {inv }}(s)}\right] \frac{1}{1+\frac{Z_{\text {grd }}(s)}{Z_{\text {inv }}(s)}}
$$

\subsection{Impedance stability criterion for photovoltaic inverter system}

The impedance analysis is based on the stability of DC steady-state operation point analysis system. In this paper, a mathematical model of inverter system is established in DQ rotating coordinate system. As follows:

$$
\left\{\begin{array}{l}
m_{d} u_{d c}=\left(L+L_{g}\right) \frac{d i_{g d}}{d t}-\omega\left(L+L_{g}\right) i_{g q}+\left(R_{L}+R_{g}\right) i_{g d}+u_{s d} \\
m_{q} u_{d c}=\left(L+L_{g}\right) \frac{d i_{g q}}{d t}+\omega\left(L+L_{g}\right) i_{g d}+\left(R_{L}+R_{g}\right) i_{g q}+u_{s q} \\
C \frac{d u_{d c}}{d t}=i_{P V}-\left(m_{d} i_{g d}+m_{q} i_{g q}\right)
\end{array}\right.
$$

The small signal analysis model of the grid-connected PV inverter system is obtained by linearizing the equation (2).

$$
\left\{\begin{array}{l}
U_{d c} \Delta m_{d}+M_{d} \Delta u_{d c}=\left(L+L_{g}\right) \frac{d \Delta i_{g d}}{d t}+\left(R_{L}+R_{g}\right) \Delta i_{g d}-\omega\left(L+L_{g}\right) \Delta i_{g q}+\Delta u_{s d} \\
U_{d c} \Delta m_{q}+M_{q} \Delta u_{d c}=\left(L+L_{g}\right) \frac{d \Delta i_{g q}}{d t}+\left(R_{L}+R_{g}\right) \Delta i_{g q}+\omega\left(L+L_{g}\right) \Delta i_{g d}+\Delta u_{s q} \\
C \frac{d \Delta u_{d c}}{d t}=\Delta i_{p V}-\left(I_{g d} \Delta m_{d}+I_{g q} \Delta m_{q}+M_{d} \Delta i_{g d}+M_{q} \Delta i_{g q}\right)
\end{array}\right.
$$

The small signal circuit of the grid-connected PV inverter system, in the dq coordinate system, can be obtained from the above figure as shown below. The input impedance of the grid side is obtained, considering the two port network on the right side of the parallel network. Based on the output impedance model of inverter controlled by PLL, the small signal transfer model of inverter under PLL control can be obtained as shown in the following figure:

$$
\begin{gathered}
Z_{g r d}=\left[\begin{array}{cc}
s L_{g}+R_{g} & -\omega L_{g} \\
\omega L_{g} & s L_{g}+R_{g}
\end{array}\right] \\
Z_{\text {inv }}=\frac{u_{g}}{i_{g}}=\left[\begin{array}{ll}
Z_{d d} & Z_{d q} \\
Z_{q d} & Z_{q q}
\end{array}\right]=\left[\begin{array}{cc}
s L+R_{L} & -\omega L \\
\omega L & s L+R_{L}
\end{array}\right]
\end{gathered}
$$

With the above figure, the output impedance model of the Grid-connected inverter is as below in consideration of the PLL and current inner loop.

$$
Z_{i n v_{-} P L L}=-\left(-Z_{i n v}^{-1}+G_{i m} G_{d e l} G_{P L L}^{m} K\right)^{-1}
$$




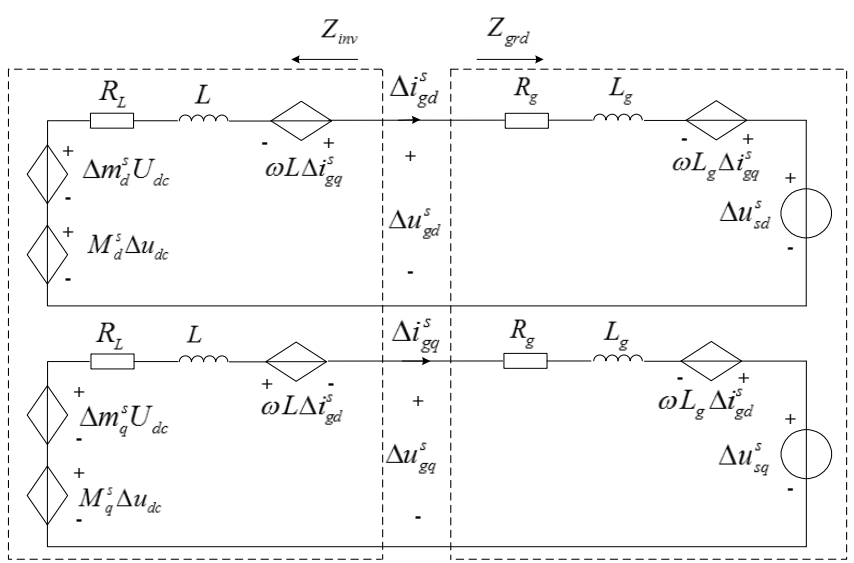

Fig. 4. The small signal circuit of inverter system in dq coordinate system

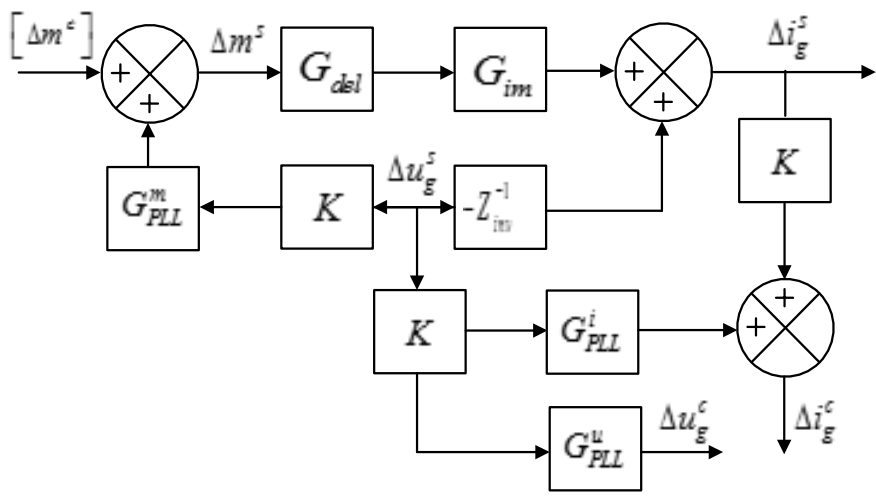

Fig.5. The small signal transfer model of inverter under PLL control

\subsection{Stability analysis of photovoltaic inverter system}

According to the output impedance of the inverter and the impedance of the grid, two sets of impedance curves under different controller parameters and the Nyquist curve of the impedance ratio can be obtained. From figure 6, it can be found that when the PLL parameters of Design1 are changed to design2, the phase difference of $\mathrm{Z}_{\mathrm{qq}}$ exceeds that of the $180^{\circ}$, which will lead to instability of the system. As can be seen from Figure 7, after the changes in the parameters of the PLL (Figure 7 (b)), the Nyquist curves contains $(-1$, j0) points, and the system will be unstable by the Nyquist stability criterion. In summary, the instability of a single inverter will affect the stability of voltage and current of the grid, and lead to the instability of other parallel inverters and systems.

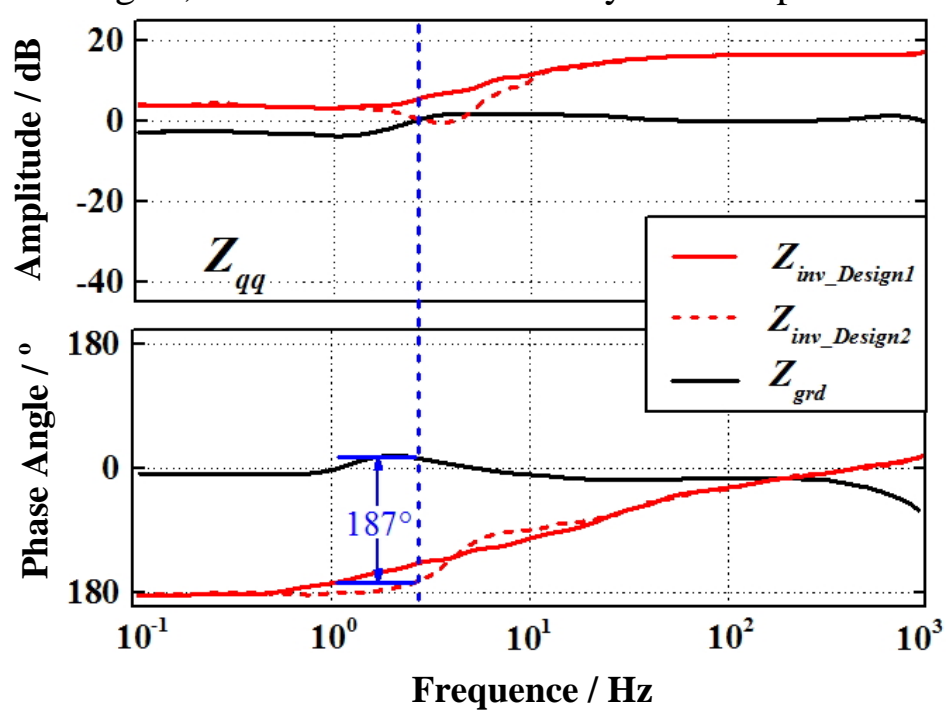

Fig.6 The impedance curves under the parameters of different phase locked loops 


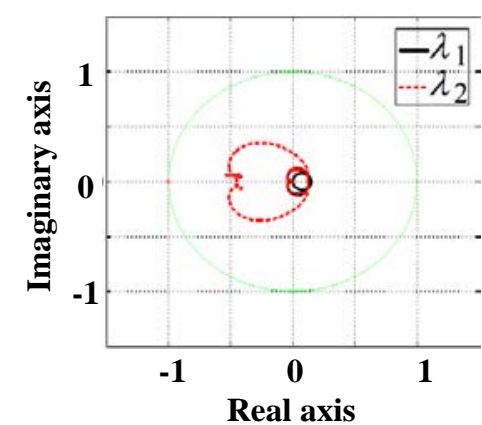

(a)The design1 parameters

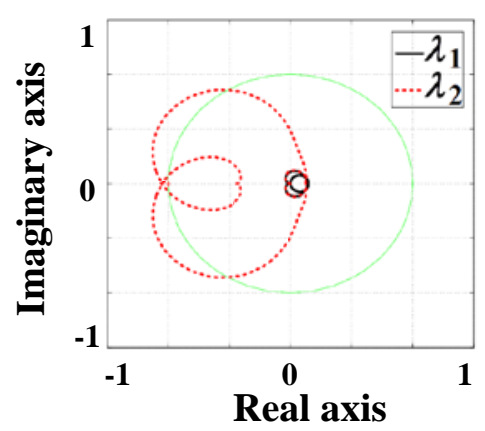

(b) The design2 parameters

Fig. 7 The Nyquist curves of the ratio of grid impedance and inverter impedance

\subsection{Stability analysis of multi photovoltaic inverter parallel system}

As the equivalent circuit of the inverter is regarded as a passive network, the equivalent impedance of the inverter can be regarded as its inherent property, that is, the equivalent impedance of a single inverter will not change by the increase or decrease of the number of parallel inverters. Therefore, the impedance analysis method is still applicable when multi photovoltaic inverters are connected to the grid in parallel.

The design of inverter phase locked loop is generally based on the ideal grid conditions. If the design of the PLL is stable, the inverter should operate synchronously and steadily under the ideal grid. After parallel inverters, the same voltage is synchronized at the common coupling point (PCC). However, under the condition of weak grid, the impedance of the grid cannot be ignored. The PCC voltage is determined not only by the grid voltage, but also by the grid impedance $\mathrm{Z}_{\mathrm{g}}$ and the injection current of the inverter. In this case, the inverter is coupled to each other because of the PCC voltage shared by the PLL. It can be seen that the instability of these PLL will make the whole system lose synchronization or even collapse. According to the above analysis, as long as the design of the inverter PLL parameters is changed, the system will keep stable.

\section{Conclusions}

In this paper, the impedance analysis method is proposed to analyze the stability of multiple inverters connected to weak grid. First, the equivalent impedance of the inverter is derived by the small signal model of the inverter in DQ coordinates, and then the stability of the inverter parallel system is analyzed according to the stability criterion. Finally, a solution to adjusting the inverter PLL is proposed to make the system stable. The simulation results verify the validity and feasibility of the theoretical analysis and the proposed method.

\section{References}

[1] Cui Rongqiang, Hang yan, Sun tietun. Difficulties and prospects of solar photovoltaic power generation in China [J]. Energy Engineering1999(5): 1-3.

[2] Zhao Chunjiang, Yang Jinhuan, Chen Zhonghua. Status and development of solar photovoltaic applications [J]. Energy Saving Technology, 2007 (5):461-465.

[3] Huang Yafeng. Research on feasible operation region of large capacity photovoltaic inverter system connected to weak grid[D]. North China Electric Power University, 2014: 36—39.

[4] C. Se-Kyo, “A phase tracking system for three phase utility interface inverters,” IEEE Trans. Power Electron., vol. 15, no. 3, pp. 431-438, May 2000.

[5] Chen Xin, Wang Chen Cheng. Research on dynamic interaction between grid connected inverter and power grid based on impedance analysis [J]. China Journal of electrical engineering, 2014. 